\title{
Effect of Intervening Bandwidth Feedback on Motor Skill Learning in Self-Controlled Feedback Context
}

\author{
Fan ZHANG ${ }^{1,2, *}$, Bo ZHOU ${ }^{1}$ and Chang-zhi $\mathrm{JIA}^{1}$ \\ ${ }^{1}$ Department of Police Skills and Tactics, Nanjing Forest Police College, Nanjing, China \\ ${ }^{2}$ Sports Science Postdoctoral Programme, Nanjing Normal University, Nanjing, China \\ ${ }^{*}$ Corresponding author
}

\begin{abstract}
Keywords: Self Control Feedback; Bandwidth Feedback; Motor Skill Learning; Motor Skill Performance.
\end{abstract}

\begin{abstract}
The feedback mechanism of self control and bandwidth feedback is inconsistent. This study integrates two feedback paradigms, taking learners' performance as the basis for feedback, and combines bandwidth feedback advantage with self-controlled feedback, to explore the additional motor learning effect of self-control feedback and to test the difference in motor performance between feedback provision and non-feedback provision. The result is as follow: Under the condition of self-controlled feedback exercise, learners require feedback to perform more accurately than those who do not require feedback. However, under the restriction of practice conditions, learners may seek feedback information through learning strategies, and produce different feedback requests in motor performance.
\end{abstract}

\section{Introduction}

Self-controlled feedback (refers to a way of feedback implementation in which people based on their own willingness to determine requirements or rejection of external feedback) is a key variable of motor learning, and forms different motor learning viewpoints, but the impact of the feedback function on motor learning has not been tested. In addition, self-controlled feedback is a way of providing feedback based on motor performance, with bandwidth feedback (a method provided by external feedback [1]. Based on the study that take pre-set error values as a basis for qualitative or quantitative feedback, when the error value of learner's motor performance falls outside the error tolerance area of the study, it provides quantitative external feedback information including error direction and value [2]. On the contrary, it provides qualitative external feedback information with correct indication). So the idea of not providing accurate feedback information for better motor performance is not quite right [3]. Therefore, it is necessary to further clarify the conflicting between these two viewpoints, and examine the additional benefits of self control and bandwidth feedback integration on motor learning in the self-controlled feedback context.

\section{Research Objects and Methods}

\subsection{Research Objects}

24 healthy Nanjing Forest Police College students were chosen as subjects (12 men and 12 women) with the average age of $20.7 \pm 1.4$. They were randomly divided into self-controlled feedback and bandwidth feedback as well as error direction misjudgment feedback group (ZD Group) and relative frequency control group (self-controlled feedback Group); each group had 10 participants (5 men and 5 women in the group).

\subsection{Research Methods}

\section{(1) Research Content}

This study focuses on the practice of self-controlled feedback, and tests its additional benefits for motor learning through the arrangement of bandwidth feedback. In addition, this study also introduces 
the bandwidth feedback factor in the self-controlled situation, discussed the motor performance change in the practice stage, and therefore proposed the following 3 research questions:

Question 1: Under the exercise conditions of self-controlled feedback, what is the effect on the motor performance when the motor error falls to a small range and provides qualitative feedback?

Question 2: Under the exercise condition of self-controlled feedback, what is the effect on motor learning when the motor error falls to the small range, provides the qualitative feedback?

Question 3: Is there any difference in the behavior of the learners' asking for feedback under the exercise conditions of self-controlled feedback?

Based on the self-controlled feedback and bandwidth feedback in motor learning, the following 3 hypotheses are proposed:

Hypothesis 1: AE and VE values of self-controlled + bandwidth feedback group's motor performance during exercise stage are significantly lower than the self-controlled feedback group.

Hypothesis 2: AE and VE values of self-controlled + bandwidth feedback group's motor performance during in the maintenance test and transfer test are significantly lower than the self-controlled feedback group.

Hypothesis 3: Learners require the $\mathrm{AE}$ value of feedback on the motor performance to be significantly below the motor without requiring feedback.

(2) Experimental Design

The subjects were randomly assigned to the ZD group and the self-controlled group. After the experiment and demonstration, the participants conducted 3 familiar exercises, and the process did not affect the results of the motor. After each practice, the results were learned in the form of data. After 3 minutes, 60 exercises were carried out in the acquisition period. On the 1th day, each group was given 60 motor exercises ( 6 sets $\times 10$ times) and the participants were given different external feedback according to the group. 24 hours after the motor exercise, the two groups maintained the test of 12 times and the transfer test of 12 times for motor execution. The test process did not provide the external feedback information.

Special Signs Self-controlled Feedback + Broadband Group (ZD Group). At the end of each practice, the learners were able to submit a request for feedback based on their own decisions. If the feedback request of the exercise performance $\mathrm{AE}$ value is lower than $30 \mathrm{~cm}$, the feedback information of "correct" for this motor performance was provided. If the error value was more than $30 \mathrm{~cm}$, then according to the result learned, the learners carried out another exercise in 10 seconds. If the learners decided not to ask for feedback this time, then the next practice started in 10 seconds.

Self-controlled Feedback Group (ZK Group). At the end of each practice, the learners were able to submit a request for feedback based on their own decisions. If the request for feedback was made, the researcher would immediately provide the results in the form of data and the next exercise started in 10 seconds. If the learners decided not to ask for feedback this time, then the next practice started in 10 seconds. After the duration of the exercise, each of the two groups kept the test of 12 times and the transfer test of 12 times. The test and transfer tests will not provide feedback during the process.

(3) Experiment.

Experimental Methods. The subjects, standing at the beginning of the platform, holding the slider in their hands, applied force to the slider at the zone of motor and release the slider in front of the screen. The extension line $300 \mathrm{~cm}$ away from the screen was used as the stop target, and the screen covering the slide area of the slider during the motor performance. At the end of each practice, the learners submitted a request for feedback based on their own decisions. If the feedback request of the exercise performance AE value is lower than $30 \mathrm{~cm}$, the feedback information of "correct" for this motor performance was provided. If the error value was more than $30 \mathrm{~cm}$, then according to the result learned, the learners carried out another exercise in 10 seconds. If the learners decided not to ask for feedback this time, then the next practice started in 10 seconds.

Experimental Principle. This experiment used intermittent power to control motor, so the result information would not be provided when the motor performers were applying force to it. The motor result can be obtained when the slider completely stopped, so the motor cannot be corrected according 
to the feedback during the motor execution, but could only be taken as the basis of the next motor, instead of correcting the performance of the current motor. The layout of the experimental equipment covers the screen. Therefore, in the absence of visual information, after the implementation of the error detection and error estimation for each motor, motor performers must make judgment based on the body feeling and dynamic awareness of internal feedback.

\section{(4) Experiment Step}

Acquisition Period. Each participant in the two groups was given a total of 60 exercises divided into 6 intervals, 10 exercises per interval, and 3 minutes between intervals.

Retention Test and Transfer Test. 24 hours after the end of the acquisition period, 12 times of exercises with the goal of $300 \mathrm{~cm}$ for the retention test was carried out as well as the transfer test with the goal of $250 \mathrm{~cm}$. No external feedback was provided during the process of retention test and transfer testing. Data was collected in order to avoid warm-up decrement. The first 2 exercises were eliminated for analysis.

Statistical Processing and Analysis. SPSS 19.0 was used for statistical processing, and the results were expressed by means- \pm standard deviation $(\bar{X} \pm \mathrm{SD})$. The independent variable provided the feedback method, because the variable was the accuracy of the motor, the stability and the error range. The accuracy of the motor is evaluated by AE, and the motor stability is evaluated by VE. The following statistical methods were proposed to validate the research hypothesis. Two factor variance analysis with the mixed design of 2 (Group) x 6 (interval) was carried out to validate hypothesis 1 , and the independent sample T-Test was adopted to analyze the AE value and VE value of retention test and transfer test to verify hypothesis 2 ; two factor variance analysis with the mixed design of 2 (feedback) $\mathrm{x} 3$ (interval) was made to verify hypothesis 3 . Statistically the significant level was $\mathrm{P}<0.05$ and the extremely significant level was for $\mathrm{P}<0.01$. For all tests, the effect size was further calculated; the variance analysis was indicated by eta square $(\eta 2)$ and Cohen's $d$ in t-Test. If the repeated measured factor violated the assumption of sphericity, it should be corrected by Greenhouse-Geisser method.

\section{Results and Analysis}

\subsection{Difference in Frequency of Feedback}

This study is set in the exercise conditions of self-controlled feedback. The feedback provided by the ZD Group is based on the learner's autonomy to provide feedback after the practice. If the AE value pf motor performance was below $30 \mathrm{~cm}$ the correct oral qualitative feedback was provided; if the AE value of motor performance was higher than $30 \mathrm{~cm}$, quantitative feedback information would be provided. For the feedback information of the self-control group, the provision was based on the learner's request to provide feedback after the practice, and all were oral quantitative feedback.

The Frequency of Qualitative Feedback for Each Interval. The self-controlled group providing qualitative feedback for the required feedback ratio was $30.6 \%$ for 1 th interval, $55 \%$ for 2 nd interval, $52.6 \%$ for 3 rd interval, $63.9 \%$ for 4 th interval, $41.6 \%$ for 5 th interval and $32.9 \%$ for 6 th interval. The request for qualitative feedback of each interval was $46.1 \%$. It was found that the interval reached statistically significant difference $(F(5,55)=7.62, p<.05, \eta 2=.37$, large effect size $)$, in which the interval 2,3 and 4 were significantly higher than the interval 1,5 and 6 . The proportion of the ZD group providing qualitative feedback in practice was $19.2 \%$ for 1 th interval, $36.7 \%$ for 2 nd interval, $41.7 \%$ for 3 rd interval, $44.2 \%$ for 4 th interval, $26.7 \%$ for 5 th interval and $19.2 \%$ for 6 th interval. The request for qualitative feedback of each interval was $31.3 \%$. It was found that statistically significant difference $(\mathrm{F}(3.31,55)=9.86, \mathrm{p}<.05, \eta 2=.41$, large effect size $)$, and the interval 3 and 4 were significantly higher than the interval 1,5 and 6 after comparison.

Frequency of Feedback Request for Each Interval. The frequency of feedback in the ZD group was $62.5 \%$ for 1 th interval, $66.7 \%$ for 2 nd interval, $73.3 \%$ for 3 rd interval, $69.2 \%$ for 4 th interval, $64.2 \%$ for 5 th interval and $58.3 \%$ for 6 th interval. The average frequency of feedback request was $65.7 \%$. The frequency of feedback in the self-control group was $60.0 \%$ for 1 th interval, $65 \%$ for 2 nd 
interval, $75 \%$ for 3 rd interval, $70.8 \%$ for 4 th interval, $69.2 \%$ for 5 th interval and $77.5 \%$ for 6 th interval. The average frequency of feedback request was $69.6 \%$. According to the two groups' feedback request frequency, after the examination, it was found that the interactive effect was of statistically significant difference $(\mathrm{F}(5,110)=3.31, \mathrm{p}<.05, \eta 2=.06$, small effect size). After comparison, it was found that in the 1 th to 5 th interval, there was no statistically significant difference between the two groups. Only in the 6th interval ZK Group, the frequency of requests for feedback was significantly higher than that of the ZD group.

\subsection{The Difference between Motor Performance and Motor Learning}

AE. Acquisition period: After examination, factor intermotor between group and interval did not reach statistically significant difference $(\mathrm{F}(2.54,110)=1.26, \mathrm{p}>.05, \eta 2=.02)$. The interval factor was of statistically significant difference $(F(2.54,110)=38.96, p<.05, \eta 2=.52$, large effect size $)$. AE value of motor performance decreased with practice in 1-5 interval. Interval 1 was significantly greater than interval 2-5; interval 2 was significantly greater than 3-6, but the AE value of interval 6 was greater than that of interval 3,4 and 5; interval 6 was significantly greater than interval 5 . The main effect of group factors was not statistically significant $(F(1,22)=.01, p>.05, \eta 2=.0)$. However, there was no significant difference between the 1th to 5 th interval, but the AE value (39.1) in the 6th interval in ZD group was significantly greater than that of the self-control group (33.6). In addition, the AE value (39.1) in the 6th interval of the ZD feedback group was significantly greater than that of interval 3 (33.2), interval 4 (32.1) and interval 5 (35.0). Retention test and transfer test: After examination, the results showed that either in the retention test $(\mathrm{t}(22)=.1 .02, \mathrm{p}>.05, \mathrm{~d}=.42$, small effect size) or in transfer test $(\mathrm{t}(22)=.99 \mathrm{p}>.05, \mathrm{~d}=.40$, small effect size), the group did not reach statistically significant differences.

VE. Acquisition period: After examination, factor intermotor of group and interval reached statistically significant difference $(F(3.67,110)=10.79, p<.05, \eta 2=.16$, large effect size $)$. The group factor reached statistically significant difference $(F(1,22)=6.71, p<.05, \eta 2=.08$, medium effect size) and the interval factor reached statistically significant difference $(F(3.67,110)=10.90, p<.05, \eta 2=.16$, large effect size). after comparison, it was found there were statistically significant differences between interval 1,2,3,5 and 6, in which the VE value of 1,2, 3 and 5 was significantly lower than that of ZK group, and in interval 6, the VE value of ZK group was significantly lower than the ZD group. However, the VE value of the 6th interval (45.0) in the ZD group was significantly higher than that of the other 5 intervals $(37.8,33.2,29.2,33.6$ and 31.1).

Retention test and transfer test: After examination, the results showed that the retention test $(\mathrm{t}(22)$ $=2.12, \mathrm{p}<.05, \mathrm{~d}=.87$, large effect size $)$ and transfer test $(\mathrm{t}(22)=2.25, \mathrm{p}<.05, \mathrm{~d}=.92$ large effect size $)$. The group was statistically significant differences. The VE value of ZD group in the retention test (32.20) was significantly lower than that of the ZK group (36.26). In the transfer test, the average VE value of ZD group (40.09) was significantly lower than the ZK group (46.09).

As mentioned above 1, there is no difference between the two groups in terms of AE, and the result did not support Hypothesis 1; in terms of VE, the result partly supports Hypothesis 1 . For the retention test and transfer test, the research results partly support Hypothesis 2, which did not support assumption 2 in the motor accuracy, but supported the Hypothesis 2 in the stability of the motor.

\subsection{Difference between the Motor Performance with and without Feedback}

The results of analyzing the actual motor of ZD group showed that there was significant difference between the feedback request and the two factor intermotor $(F(1.56,44)=27.78, p<.05, \eta 2=.20$ large effect size), and the feedback request factor reached statistically significant difference $(\mathrm{F}(1,22)=$ 27.13, $\mathrm{p}<.05, \eta 2=.08$, medium effect size). The interval factor reached statistically significant difference $(\mathrm{F}(1.56,44)=15.81, \mathrm{p}<.05, \eta 2=.11$, medium effect size $)$. The interval 1 was significantly greater than interval 2 and interval 3; there was no significant difference between interval 2 and interval 3 (AE values in the interval were 52.0, 39.9 and 35.3, respectively). After the comparison, it was found that the $\mathrm{AE}$ values were significantly different in the 3 intervals, which required feedback. 
The AE value of interval 1 and interval 2 requiring feedback was significantly lower than that not requiring feedback, while in interval 3 the $A E$ value of interval not requiring feedback was significantly lower than that of requiring feedback. (AE value of intervals requesting feedback was 37.8, 29.0 and 42.3 respectively; AE value of intervals not requesting feedback was 66.3, 50.7 and 28.3 respectively).

For the AE value analysis of the actual motor performance in $\mathrm{ZK}$ group, the intermotor between feedback request and the two interval factors did not reach statistically significant difference $(\mathrm{F}(2,44)$ $=1.96, \mathrm{p}>.05, \eta 2=.01)$. The feedback request reached statistically significant difference $(\mathrm{F}(1,22)=$ 55.77, $\mathrm{p}<.05, \eta 2=.26$ large effect size). The $\mathrm{AE}$ value of motor performance with feedback request was significantly lower than that without requesting feedback (The AE value of motor performance with feedback request was $38.1,28.3$ and 30.0 respectively; the $\mathrm{AE}$ value of motor performance without feedback request was $62.6,47.1$ and 43.9 , respectively.). The interval factor was significantly different $(\mathrm{F}(2,44)=15.64$, p\&lt;.05, $\eta 2=.11$ effect amount $)$. The interval 1 was significantly lower than interval 2 and interval 3; interval 2 and interval 3 had no significant difference (AE values in each interval were 50.3, 37.7 and 37.0 , respectively)

The results of the study in the ZK Group partly supported Hypothesis 3 and the ZD group partly supported Hypothesis 3. From the two groups' response to the accuracy of the performance, it can be found that in the self-controlled feedback context, the 3 interval of ZK group in the acquisition period and the first 2 intervals of the ZD group was not as accurate as that without requesting feedback, which was consistent with previous studies.

\section{Summary}

In the exercise of self-controlled feedback combined with bandwidth feedback, when the error of $\mathrm{AE}$ value for motor performance falls in a small range after the provision of qualitative feedback information, there is no positive impact on the accuracy of the motor performance, but for the stability of the motor performance, it has a role in promoting. In the exercise of self-controlled feedback combined with bandwidth feedback, when the error of AE value for motor performance falls in a small range after the provision of qualitative feedback information, there is no positive impact on the accuracy of the motor learning, but for the stability of the motor learning, it has a role in promoting. Under the condition of self-controlled feedback exercise, learners require feedback to perform more accurately than those who do not require feedback. However, under the restriction of practice conditions, learners may seek feedback information through learning strategies, and produce different feedback requests in motor performance.

\section{Acknowledgement}

This work was financially supported in part by the Project of the Fundamental Research Funds for the Central Universities under Grant LGZD201805, and in part by Jiangsu University Philosophy and Social Sciences Research Fund Project under Grant 2017SJB0590.

\section{References}

[1] Wulf G, Shea C, Lewthwaite R, Motor skill learning and performance: a review of influential factors [J]. Medical Education, 2010, 44 (1): 75-84.

[2] Schmidt R A, Lee T D. Motor control and learning: a behavioral emphasis [M]. Human Kinetics, 1999.

[3] Sadowski J, Mastalerz A, Niznikowski T. Benefits of bandwidth feedback in learning a complex gymnastic skill [J]. Journal of Human Kinetics, 2013, 37 (1): 183-193. 